\title{
Isolated Ventricular Non-Compaction Syndrome, Rare Cause of Recurrent Stroke in Young - A Case Report
}

\author{
Rima Rumpaul Khanna ${ }^{1}$, Rajesh Garg${ }^{1}$, Hemant Madan², Neeraj Mahajan² and Pankaj Kumar ${ }^{3}$ \\ ${ }^{1}$ Department of Neurology, Fortis Hospital, Shalimar Bagh, New Delhi, India \\ ${ }^{2}$ Department of Cardiology, Fortis Hospital, Shalimar Bagh, New Delhi, India \\ ${ }^{3}$ Department of Radiology, Fortis Hospital, Shalimar Bagh, New Delhi, India
}

Corresponding author: Rima R Khanna, Associate Consultant, Department of Neurology, Fortis Hospital, Shalimar Bagh, New Delhi, India, Tel: 011 45151695; 8130840101; E-mail: rumpaulrima@gmail.com

Received: Mar 30, 2016; Accepted: May 06, 2016; Published: May 10, 2016

\begin{abstract}
Strokes in young adults are uncommon and often a diagnostic challenge. It can cause morbidity, motality and hamper quality of life. A cardiogenic cerebral embolus is one of the most common causes of stroke in the young, accounting for up to one third of the cases. In this article we describe a rare case of isolated left ventricle non compaction as an etiology for recurrent strokes in young individuals. Echocardiography often provides the first clue to diagnose
\end{abstract}

Furthermore, lifelong systemic anticoagulation is indicated to obviate risk for thromboembolism and reduce the recurrences of further morbid states.

Keywords: Recurrent strokes; Non-compaction; Left ventricle; Cardioembolic; Cardiomyopathy

Abbreviations: CMRI-Cardiac Magnetic Resonance Imaging; LVNC-Left Ventricle Non Compaction; AF-Atrial Fibrillation; LV-Left Ventricle; IVNC-Isolated Left Ventricular Non-Compaction Cardiomyopathy; MRAMagnetic Resonance Angiography; DWI-Diffusion Weighted Imaging

\section{Introduction}

Strokes in young adults are uncommon and often a diagnostic challenge. According to available data, less than $5 \%$ of all strokes occur in subjects under 45 years of age, in developed countries [1] and between 19-30\%, in developing countries $[2,3]$. The incidence of stroke in young adults is higher in developing countries because of the higher incidence of infections, rheumatic heart disease and undetected or uncontrolled vascular risk factors [4-7].

Conventional risk factors for stroke are not frequent in young age. Young patients have special risk factors inherent to genetic and environmental elements, such as cardiac abnormalities, thrombophyllic states, migraine, use of oral contraceptives and illicit drugs that should be identified and adequately controlled [8]. The main clinical challenge in management of a young adult with acute stroke is the identification of its cause. In the Helsinki Young Stroke Registry 2 (1008 patients aged $<50$ years), cardioembolism (20\%) and cervicocerebral artery dissection (15\%) were the most frequent aetiological subgroups [9]. In young individuals, highrisk sources of embolism detected by echocardiography include mechanical prosthetic valves, mitral stenosis, endocarditis (infective and non-infective) [10,11] dilated cardiomyopathies, intracardiac thrombus, and cardiac tumours such as myxoma and fibroelastoma [12]. The most common uncertain sources of embolism are patent foramen ovale and atrial septal aneurysm, akinetic or dyskinetic segments of the ventricular wall and redundant mitral valve prolapse.

In this article we describe a rare case of isolated left ventricle non compaction as an etiology for recurrent strokes in young individuals.

\section{Case}

A 24 year old gentleman presented with multiple episodes of focal neurological deficits for last 3 months. Initially he had 5 episodes of right upper limb weakness which persisted for few minutes and subsequently had complete recovery of symptoms. Later he developed 7-8 episodes of weakness of right lower limb, had difficulty in walking for few minutes followed by complete recovery. In December last year he had couple of episodes of motor aphasia which alarmed him and brought him to medical attention. There was no significant past history of migraine, alcohol and drug abuse. He had no family history of cardiomyopathy or sudden death.

On examination he was a well-built individual with normal blood pressure, heart rate and rhythm. Neurologically he was alert with no focal neurological deficit. His MRI (Brain) showed multiple patchy areas of diffusion restriction seen in bilateral frontal lobes, right temporal lobe and right caudate nucleus suggestive of acute infarcts and an area of encephalomalacia in right parietal lobe (Figure 1). On further evaluation his ECG, MRA (Brain and Neck), procoagulant profile, vasculitis profile and $24 \mathrm{hrs}$ holter monitoring were normal. Echocardiography was suggestive of focal area trabeculation in apex of left ventricle with blood flowing in and out of it (Figure 2). Further patient underwent Cardiac MRI, which revealed fine hypertrabeculation of left ventricle in apical region more 
towards the lateral wall with ratio of non-compacted myocardium to normal myocardium $>2$ suggestive of noncompaction cardiomyopathy (Figure 3 ). Thus he was started on oral anticoagulant so as to keep the INR in between 2-3. Thereafter he had no recurrence of any focal neurological deficit and was tolerating medicine well.

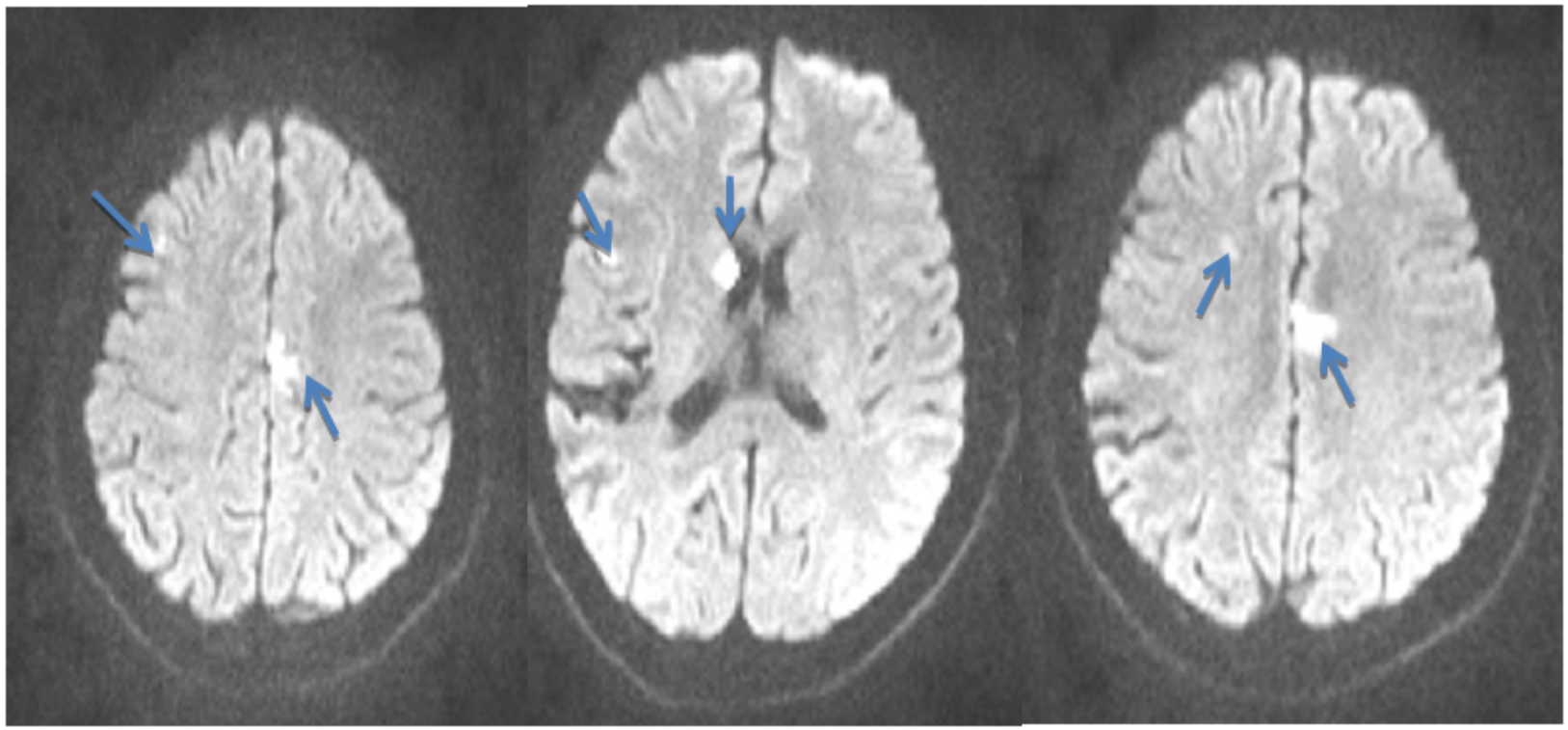

Figure $1 \mathrm{MRI}$ (Brain, Axial DWI images) showing restriction of diffusions in multiple areas.

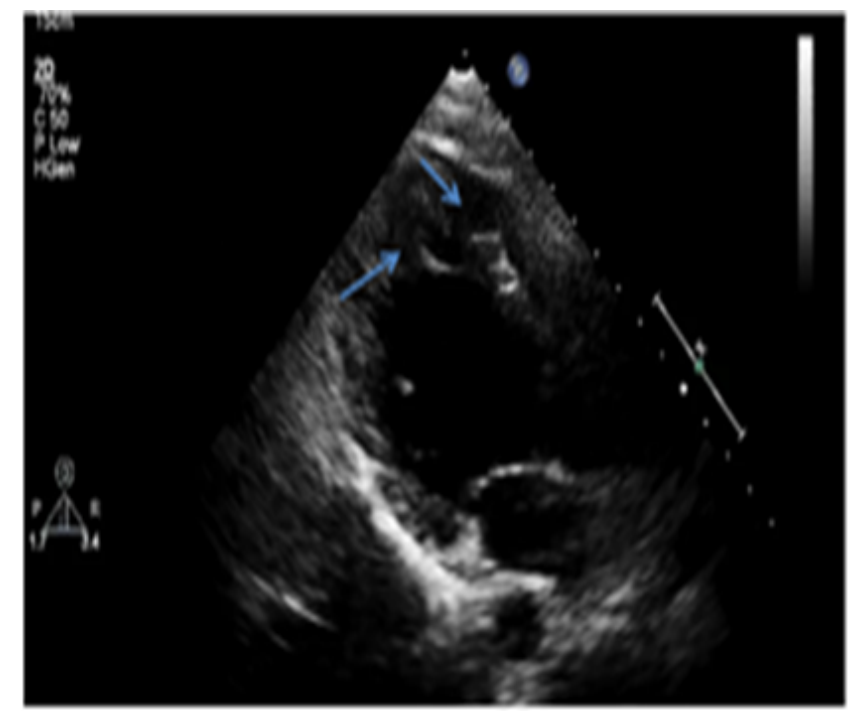

Figure 2 2D-Echo images showing out pouching in the left ventricular apex.

\section{Discussion}

In this era when the diagnosis and treatment of stroke has shown dramatic improvement, ischemic stroke in young adults remains a catastrophic event. It causes mortality, significant morbidity and hampers their quality of life. From the prospective of a neurologist, the main challenge is to identify its risk factors and etiology so as to prevent its recurrence. Neuroimaging of the brain, its vessels, a comprehensive cardiac assessment and thrombophyllic profile will enable us to identify the most frequent causes of stroke in young adults. Despite extensive evaluation, the etiology remains elusive in majority of studies leading to recurrences and increased morbidity.

A young male with no conventional risk factors, no high risk behaviour, no significant family history and with a healthy lifestyle of regular exercise and diet, presented with recurrent episodes of focal neurological deficits causing him significant mental and physical trauma. He was extensively evaluated for delineating the cause of his recurrent events with conventional investigations but all investigations were normal except carefully done echocardiography.

The frequency of recurrent strokes in the young is lower as compared with older patients. The cumulative recurrence rate at 5 years is almost 2 times lower in young (15\%) than in older $(29.5 \%)$ patients $[13,14]$. The recurrence rate is higher in the first year (3\% to $5 \%)$ and decreases in subsequent years $(2 \%$ to $5 \%)$. Recurrence is more frequent in patients with atherothrombotic stroke (about 5\% annual) than in those with stroke due to non-atherosclerotic vasculopathy (about 2\%) [13]. This young individual had multiple episodes of stroke over a course of few weeks which is quite contrary to the available literature. The recurrences were embolic in nature, arising from heart probably due to hypertrabeculations in the left ventricle. During his stay in the hospital we were not able to detect any episode of atrial fibrillation. Although a cardiogenic cerebral embolus is one of the most common causes of stroke in the young, accounting for up to one third of the cases $[15,16]$ but this entity isolated left ventricular non-compaction cardiomyopathy (LVNC) is not included in the etiological lists of young stroke. 


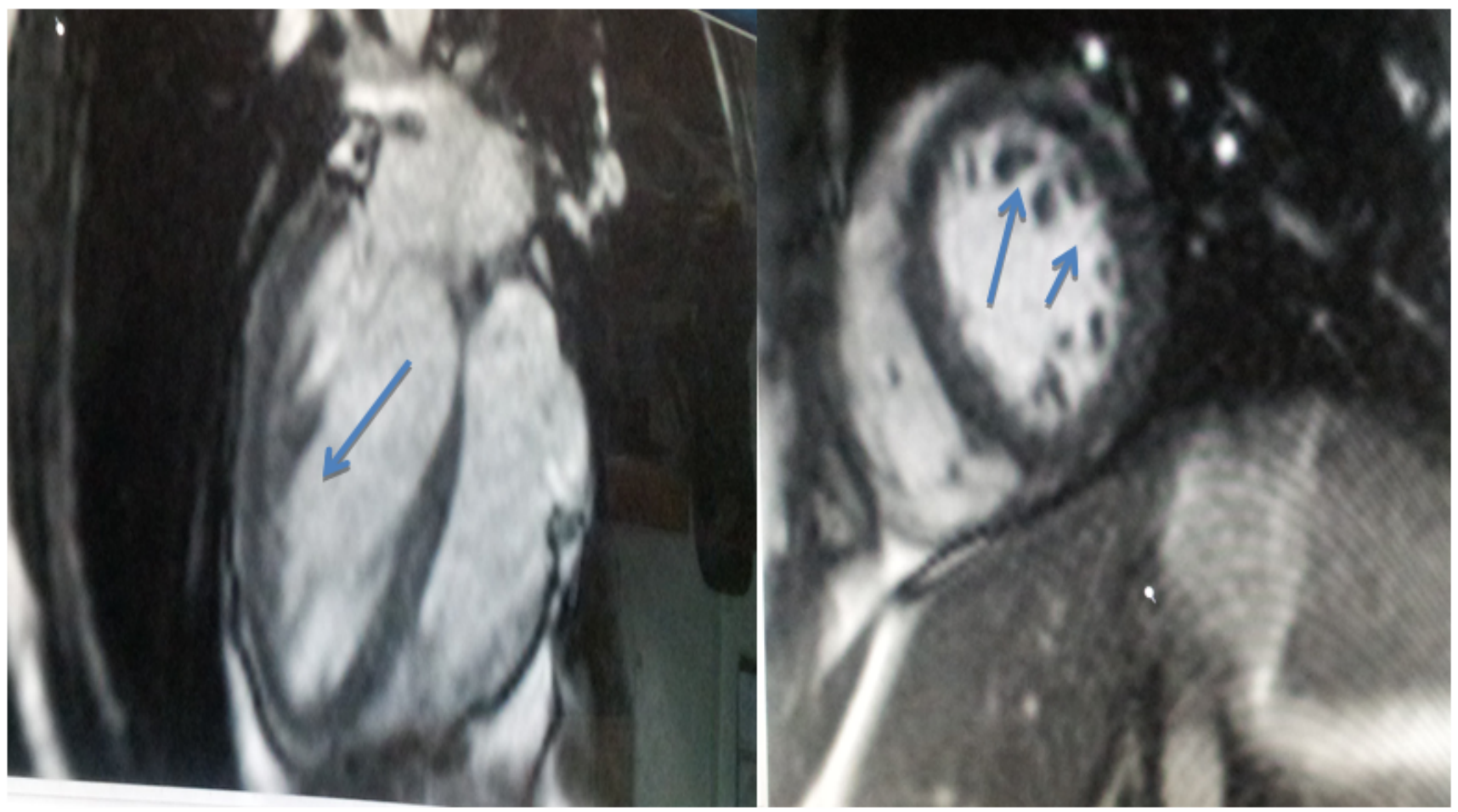

Figure 3 Cardiac MRI showing multiple trabeculations in left ventricle.

Isolated left ventricular non-compaction cardiomyopathy (IVNC) is a rare, morphologically distinct primary genetic cardiomyopathy with first clinical description in 1984 by Engberding. It is a relatively rare congenital disorder and is a rarer cause of stroke in young.

Most authors believe in the congenital theory which suggests that it occurs in-utero when the segments of spongy myocardium fail to transform into compact, mature musculature thus resulting in prominent myocardial trabeculae and deep intra-trabecular recesses. However this hypothesis lacks proof. Its prevalence is extremely rare in young adults, less than $0.3 \%$ [14]. The clinical presentations of LVNC include heart failure, arrhythmias, and propensity for thromboembolism (21 to 24\%). The increased risk for thromboembolism is believed to be multi-factorial; and is due to the combination of impaired LV function, perhaps simultaneous occurrence of atrial fibrillation, and the presence of abnormal myocardial trabeculae. Echocardiography often provides the first clue to diagnose LVNC which is then confirmed with Cardiac Computed Tomography and cardiac MRI.

Once diagnosed it requires lifelong systemic anticoagulation so as to obviate risk for thromboembolism and reduce the recurrence of further morbid states.

We have come across quite a few case reports of LVNC with stroke but none with the recurrent episodes of stroke over a course of days. There have been fewer reported cases of peripheral cardiac embolic complications but that were associated with LV dysfunction and arrthymias. In our patient's case we were not able to find any evidence of AF and left ventricular dysfunction. Although we were also not able to demonstrate thrombus formation within the intertrabecular recesses by Echocardiography and CMRI, we postulated that the source of emboli was abnormal trabeculations seen in the left ventricle which was the only abnormal finding in this patient.

\section{Conclusion}

LVNC is a rare and treatable cause of stroke. If detected early, the risk of morbidity and mortality in otherwise healthy young adults from stroke can be reduced by anticoagulation. We would like to increase the awareness about left ventricular non-compaction cardiomyopathy as one of the rare causes of stroke in young adults and its diagnosis with carefully done echocardiography and cardiac MRI .We also suggest Left ventricular non-compaction cardiomyopathy should be included in the list of rare causes of stroke in young adults.

\section{Conflict of Interest}

This is non-funded and non-sponsored study. The authors declare that they have no conflict of interest.

\section{References}

1. Nencini P, Inzitari D, Baruffietal MC (1998) Incidence of stroke in young adults in Florence, Italy. Stroke 19: 977-981.

2. Radhakrishnan K, Ashok PP, Sridharan R, Mousa ME (1986) Stroke in the young: incidence and pattern in Benghazi, Libya. Acta Neurologica Scandinavica 73: 434- 438. 
3. Kittner SJ, McCarter RJ, Sherwin RW (1993) Black-white differences in stroke risk among young adults. Stroke 24: I13115.

4. Lipska K, Sylaja PN, Sarma PS (2007) Risk factors for acute ischemic stroke in young adults in South India.J Neurol Neurosurg Psychiatry 78: 959-963.

5. Mehndiratta MM, Agarwal P, Sen K, Sharma B (2004) Stroke in young adults: a study from a university hospital in North India.Med Sci Monit 10: 535-541.

6. Dharmasaroja PA, Muengtaweepongsa S, Lechawanich C, Pattaraarchachai J (2010) Causes of ischemic stroke in youngadults in Thailand: a pilot study. J Stroke Cerebrovasc Dis 20: $247-250$

7. Samiullah S, Humaira M, Hanif G, Ghouri AA, Shaikh K (2010) Etiological patterns of stroke in young patients at a tertiary care hospital. J Pak Med Assoc 60: 201-204.

8. Marini C, Totaro R, Carolei A (1999) Long-term prognosis of cerebral ischemia in young adults. Stroke 30: 2320-2325.

9. Jukka Putaala, Metso AJ, Metso TM, Konkola N, Tatlisumak T (2009) Analysis of 1008 consecutive patients aged 15 to 49 with first ever ischemic stroke: The Helsinki young stroke registry . Stroke 40: 1195-1203.

10. Huang TY, Tseng HK, Liu CP, Lee CM (2009) Comparison of the clinical manifestations of infective endocarditis between elderly and young patients-a 3-year study. J Microbiol Immunol Infect 42: 154-159.

11. Nishimura RA, Carabello BA, Faxon DP (2008) ACC/AHA 2008 Guideline update on valvular heart disease: focused update on infective endocarditis: A report of the American College of Cardiology/American Heart Association Task Force on Practice Guidelines endorsed by the Society of Cardiovascular Anesthesiologists, Society for Cardiovascular Angiography and Interventions, and Society of Thoracic Surgeons. J Am Coll Cardiol 52: 676-685.

12. Egouskov V, Kadner A, Engelberger L, Carrel T, Tevaearai H (2008) Papillary fibroelastoma of the heart. Heart Surg Forum 11: 333-339.

13. Varona JF, Bermejo F, Guerra JM, Molina JA (2004) Long- term prognosis of ischemic stroke in young adults: study of 272 cases. Journal of Neurology 251: 1507- 1514.

14. Burn J, Dennis M, Bamford J, Sandercock P, Wade D, et al. (1994) Long-term risk of recurrent stroke after a first-ever stroke: the Oxfordshire community stroke project. Stroke 25: 333-337.

15. Hart RG, Freeman GL (1987) Stroke in young people-The heart of the matter. West J Med 146: 596-597.

16. Hachinslri V, Norris JW (1985) The young stroke- in the acute stroke. Contemporary Neurology Series 27: 141-163. 